Relations industrielles

Industrial Relations

\title{
D. Ashton and G. Lowe, Making thier Way: Education, Training and the Labour Market in Canada and Britain
}

\section{Clément Lemelin}

Volume 46, numéro 4, 1991

URI : https://id.erudit.org/iderudit/050733ar

DOI : https://doi.org/10.7202/050733ar

Aller au sommaire du numéro

Éditeur(s)

Département des relations industrielles de l'Université Laval

ISSN

0034-379X (imprimé)

1703-8138 (numérique)

Découvrir la revue

Citer ce compte rendu

Lemelin, C. (1991). Compte rendu de [D. Ashton and G. Lowe, Making thier Way: Education, Training and the Labour Market in Canada and Britain]. Relations industrielles / Industrial Relations, 46(4), 881-883.

https://doi.org/10.7202/050733ar

Tous droits réservés (C) Département des relations industrielles de l'Université Laval, 1991
Ce document est protégé par la loi sur le droit d'auteur. L’utilisation des services d'Érudit (y compris la reproduction) est assujettie à sa politique d'utilisation que vous pouvez consulter en ligne.

https://apropos.erudit.org/fr/usagers/politique-dutilisation/ 
Making their Way: Education, Training and the Labour Market in Canada and Britain, edited by D. Ashton and G. Lowe, Toronto, University of Toronto Press, 1991, 219 p. ISBN: 0-8082-6823-5 (paper); 0-8020-2773-3 (cloth)

Ce volume, à la rédaction duquel ont participé neuf chercheurs anglais, canadiens et allemand, porte sur l'insertion des jeunes dans le marché du travail au Canada et en GrandeBretagne. On y met l'accent sur l'emploi plutôt que sur le chômage et on s'intéresse au rôle joué par le système d'enseignement et diverses autres institutions visant à assurer la formation de la main-d'oeuvre. En utilisant une approche comparative, on espère montrer l'influence des institutions et des valeurs sur les conditions de vie.

Il comprend dix chapitres, dont une introduction et une conclusion par les co-éditeurs. Deux chapitres décrivent l'évolution récente du marché du travail dans les deux pays: alors qu'en Grande-Bretagne, la demande des jeunes de 16 ans sur le marché du travail s'est écroulée (est-ce là une autre manifestation de la sclérose européenne?), au Canada s'est développé un véritable sous-marché du travail des jeunes, dont plusieurs semblent avoir de la difficulté à se dégager. Ainsi les problèmes des jeunes apparaissent différents: chômage dans le premier cas et sous-utilisation des compétences dans le deuxième.

Deux chapitres portent sur les systèmes d'éducation de la Grande-Bretagne et du Canada et deux autres utilisent des données longitudinales pour décrire le passage de l'école au travail dans les deux pays. Un chapitre analyse les valeurs des décrocheurs de l'école secondaire de la ville d'Edmonton et un dernier fait la critique de l'ensemble des contributions et s'interroge sur la pertinence de l'approche comparative retenue.

Making their Way est le fruit d'un projet qui a permis aux divers auteurs de se réunir lors d'une conférence pour discuter de leurs résultats. Le lecteur peut se féliciter d'une telle initiative et, présumément, du travail de synthèse des deux co-éditeurs: ce volume n'a rien de désarticulé et l'on trouve dans la plupart des textes des références à la situation dans l'autre pays ainsi qu'un nombre de références aux autres textes plus considérable que ce à quoi nous ont habitués de tels ouvrages collectifs. Cela dit, la dimension comparée de ce projet de recherche reste limitée: la même stratégie de recherche n'est pas toujours utilisée pour les deux pays, pas plus que les données ne sont toujours directement comparables.

Les transitions entre l'école et le travail paraissent on ne peut plus différentes. Elle est hâtive et abrupte en Grande-Bretagne: dès qu'ils en ont la chance [...], la majorité des jeunes britanniques quittent l'école. Elle est également devenue très diffícile au début des années 1980. En comparaison, cette transition apparaît comme un processus lent et prolongé au Canada: cette impression est accentuée par le fait qu'une importante proportion des élèves canadiens travaille, certes pendant l'été, mais également pendant l'année scolaire, à temps partiel. Elle y est aussi un processus plus complexe: bon nombre de jeunes étudient à temps partiel et les mouvements ne sont pas toujours définitifs, se faisant des études vers le travail et inversement.

Le rôle de l'école dans la préparation au travail est beaucoup plus important au Canada qu'en Grande-Bretagne. Pour tout dire, alors qu'au Canada on a tendance à se reposer sur le système d'enseignement, caractérisé par la diversité des programmes et la multiplicité des cours, pour assurer aux jeunes un minimum de compétences professionnelles, en GrandeBretagne cette tâche revient dans bien des cas aux entreprises et, dans une moindre mesure, aux syndicats qui forment ainsi sur le tas; les programmes d'apprentissage y ont jusqu'à très récemment joué un rôle beaucoup plus considérable qu'au Canada.

La stratégie britannique n'a de sens que si les jeunes peuvent trouver à la sortie de l'école un emploi qui leur permet d'acquérir une formation sur le tas. La crise du début des années 
1980 a fait disparaître la plupart de ces emplois d'entrée, menant à un véritable effrondrement du marché du travail des jeunes. Aussi est-il étonnant de découvrir que les jeunes britanniques sont toujours très nombreux à quitter l'école à l'âge minimum, c'est-à-dire 16 ans.

Pour expliquer ce comportement, bien des auteurs de ce volume font référence aux valeurs des jeunes britanniques, ce qui ne saurait surprendre de la part d'un groupe constitué en grande majorité de sociologues. Ainsi il existerait une éthique britannique du travail qui engendrerait le besoin d'obtenir un emploi le plus rapidement possible; dans les milieux ouvriers, cette éthique prendrait une forme virulente et anti-scolaire, décrite par Paul Willis dans Learning to Labour. À plusieurs reprises, le système britannique d'enseignement est décrit comme sélectif et orienté vers les besoins des classes moyennes. Écrivant sur le même sujet, un groupe d'économistes aurait sans doute insisté davantage sur la difficulté de financer les études et, notamment, le manque à gagner, chez les jeunes de 16 à 18 ans en Grande-Bretagne.

Le système canadien d'enseignement apparaît très différent. L'égalité des chances en éducation est un idéal auquel la majorité souscrit. Pour assurer la démocratisation de l'école et éviter de rebuter les jeunes, on a procédé à une diversification visant à permettre aux jeunes de prolonger leurs études le plus longtemps possible. Ainsi le système d'enseignement serait l'objet d'un préjugé favorable pour ainsi dire universel: employeurs, employés et jeunes accordent tous de l'importance aux titres scolaires.

C'est ainsi qu'à la lecture de ce volume s'esquisse petit à petit une comparaison de deux systèmes d'enseignement qui est très favorable au Canada. En Grande-Bretagne, le système scolaire est rigide, sélectif et s'accompagne d'un taux de fréquentation très faible: il vise avant tout à assurer la reproduction d'une petite élite et achemine vers le marché du travail une masse peu qualifiée. Au Canada, on observe plutôt un système diversifié, flexible et démocratique: tout est mis en place pour encourager les jeunes à prolonger leurs études le plus longtemps et les taux élevés de fréquentation semblent indiquer que l'objectif d'égalité des chances est mieux atteint.

Si l'on ajoute à ces descriptions le fait que le problème de l'insertion des jeunes est beaucoup plus aigu en Grande-Bretagne qu'au Canada, on peut se demander ce qu'un Canadien préoccupé de réformes pourrait retenir d'utile d'une comparaison des systèmes britannique et canadien. Avant de conclure que le système canadien est éminemment supérieur, on notera d'abord qu'il est beaucoup plus coûteux, ainsi que le montre toute comparaison des indicateurs d'effort en éducation.

De plus, il ne va pas sans poser des problèmes auxquels il est fait allusion dans ce volume. À la lecture de plusieurs des chapitres, il ressort en effet que ce sont les lettres de créance beaucoup plus que le contenu des études qui souvent importent. D'où l'accent sur la quantité (fréquentation, durée de la scolarisation) beaucoup plus que sur la qualité des études (contenu des études, rigueur de la formation, pertinence, etc.) et une conception étroitement instrumentale de l'éducation. Si la pertinence des études et la constitution des bases (sciences, mathématiques, langues) importent peu, il faut s'interroger davantage sur le rôle de l'école dans la préparation au travail et se demander si d'autres institutions ne seraient pas mieux adaptées pour préparer les jeunes au marché du travail.

Enfin, et ainsi que le suggère Walter Heinz au chapitre 9, tout compte fait, les stratégies de formation de la main-d'oeuvre de la Grande-Bretagne et du Canada ne sont peut-être pas si différentes, puisqu'elles accordent toutes deux un grand rôle à l'expérience. Au Canada, la sélection à l'entrée se fait certes selon l'éducation mais les promotions sont attribuées selon l'expérience; pour la majorité des travailleurs britanniques l'éducation a moins d'importance, mais l'essentiel de la formation s'acquiert sur le tas. Ces deux stratégies diffèrent radicalement 
d'autres, comme celle de l'Allemagne, qui apparaît beaucoup plus structurée, avec son mélange de formation scolaire et professionnelle, dispensée conjointement par l'école et l'industrie. Aussi ce volume nous rappelle-t-il que nous aurions probablement tout autant a apprendre d'une comparaison avec le système allemand!

Clément Lemelin

Université du Québec à Montréal

Legislating Pay Equity to Raise Women's Wages. A Progress Report on the Implementation of the Ontario, Canada Pay Equity Act, National Committee on Pay Equity, Washington, D.C., $1990,122 \mathrm{p}$.

Il est intéressant de constater que l'expérience d'équité salariale d'une province canadienne, en l'occurrence l'Ontario, fasse l'objet d'autant d'intérêt chez nos voisins du Sud. Cette récente publication du National Committee on Pay Equity (NCPE) consacre, $s$ 'il en était besoin, l'avance considérable prise par l'Ontario dans l'application du principe de l'équité salariale. Ce rapport vise une diffusion la plus large possible de l'expérience de l'Ontario et veut offrir aux praticiens et aux praticiennes l'opportunité de réfléchir sur leur expérience.

Après avoir établi une liste chronologique des dates les plus importantes dans la courte histoire de la loi ontarienne sur l'équité salariale, on en présente les principales dispositions qui touchent le processus d'implantation dans les entreprises (employeurs couverts, identification des classes d'emplois, choix d'un système neutre de comparaison des emplois, cueillette des informations sur les emplois, échéancier s'appliquant aux entreprises, ajustements salariaux, etc.).

La section suivante présente les organismes créés par la loi pour voir à son administration et à son application. Il s'agit essentiellement de la Commission de l'équité salariale, elle-même constituée de deux organismes différents: le bureau et le tribunal. Quelques exemples de décisions rendues par le service de révision du bureau et par le tribunal nous aident à bien comprendre la spécificité de ces deux organismes.

Le rapport se poursuit en laissant la parole à différents groupes impliqués dans l'implantation de l'équité: syndicats, associations, coalition sur l'équité salariale, associations d'employeurs, etc. Cette section illustre certains écueils rencontrés par ces groupes dans la phase d'implantation de l'équité. On y retrouve aussi plusieurs critiques fréquemment adressées à la loi. Cette section est suivie d'une description de quelques résultats d'expériences d'équité salariale touchant le secteur public syndiqué, les municipalités et le secteur privé syndiqué et non syndiqué.

Le rapport se termine en identifiant les critiques les plus souvent adressées à la loi ontarienne et les amendements revendiqués par la Commission de l'équité salariale et d'autres groupes d'intérêt. Enfin, on retrouve en annexe du matériel d'information supplémentaire provenant de la Commission.

Comme on a pu le constater, le rapport essaie de couvrir l'ensemble des aspects les plus connus de l'expérience ontarienne. Pour ce faire, il puise abondamment aux documents déjà publiés par la Commission de l'équité salariale, par des syndicats ou d'autres groupes d'intérêt. On n'y retrouve pas de matériel original ni de réflexion approfondie sur le type d'équité véhiculé par la loi. Il s'agit véritablement d'un rapport d'étape dans lequel on soulève des questions très pertinentes en nous laissant cependant sur notre appétit car on s'empresse de préciser que l'expérience ontarienne est encore trop récente pour qu'on puisse y répondre. 